assays on the Roche LightCycler. Lab Invest. 2004;84:1200-8. http://dx.doi.org/10.1038/labinvest.3700143

8. Hammarlund E, Lewis MW, Carter SV, Amanna I, Hansen SG, Strelow LI, et al. Multiple diagnostic techniques identify previously vaccinated individuals with protective immunity against monkeypox. Nat Med. 2005;11:1005-11. http://dx.doi.org/10.1038/nm1273

9. Lopera JG, Falendysz EA, Rocke TE, Osorio JE. Attenuation of monkeypox virus by deletion of genomic regions. Virology. 2015;475:129-38. http://dx.doi.org/10.1016/j.virol.2014.11.009

10. Peters JC. An epizootic of monkey pox at Rotterdam Zoo. Int Zoo Yearb. 1966;6:274-5. http://dx.doi.org/10.1111/j.1748-1090.1966. tb01794.x

Address for correspondence: Wenjie Tan, Chinese Center for Disease Control and Prevention, 155 Changbai Rd, Changping District, Beijing 102206, China; email: tanwj28@163.com

\section{Leishmaniasis in Norway Rats in Sewers, Barcelona, Spain}

\author{
Maria Teresa Galán-Puchades, \\ Mercedes Gómez-Samblás, \\ Jose M. Suárez-Morán, Antonio Osuna, \\ Joan Sanxis-Furió, Jordi Pascual, \\ Rubén Bueno-Marí, Sandra Franco, \\ Víctor Peracho, Tomás Montalvo, Màrius V. Fuentes
}

Author affiliations: Universitat de València, Burjassot-Valencia, Spain (M.T. Galán-Puchades, J. Sanxis-Furió, M.V. Fuentes); Universidad de Granada, Granada, Spain (M. Gómez-Samblás, J.M. Suárez-Morán, A. Osuna); Laboratorios Lokímica, Catarroja-Valencia, Spain (J. Sanxis-Furió, R. Bueno-Marí); Agència de Salut Pública de Barcelona, Barcelona, Spain (J. Pascual, S. Franco, V. Peracho, T. Montalvo); CIBER Epidemiology and Public Health, Barcelona (T. Montalvo)

DOI: https://doi.org/10.3201/eid2506.181027

We detected Leishmania infantum in 98 Norway rats (Rattus norvegicus) trapped in parks and sewers of Barcelona, Spain. The 84 rats from the sewers showed a prevalence of $33.3 \%$ and up to 2,272 estimated parasites. These results, in the most abundant potential reservoir in cities, is of public health concern.

$\mathrm{C}$ Tanine and human leishmaniasis caused by Leishmania infantum is considered an emerging disease in the Mediterranean basin (1). In addition to dogs, several wild mammals have been found infected by L. infantum in rural environments in Europe (2). With regard to the epidemiologic factors promoting infection with Leishmania, the appearance of new animal reservoirs besides dogs has been highlighted (1). In this context, only a few studies examine the possible reservoir role of synanthropic animals in cities, where the role of certain domestic mammals has been analyzed exclusively (1).

Because human leishmaniasis is endemic in Barcelona, Spain (3), we investigated and quantified the presence of $L$. infantum in an urban population of the Norway rat, Rattus norvegicus, using a highly sensitive quantitative PCR (qPCR) method for Leishmania DNA detection. Rat leishmaniasis could complicate the epidemiologic situation of human and canine leishmaniasis, considering that the Norway rat is the most widespread mammal in the world after humans and also the most abundant animal in cities.

We trapped 98 Norway rats, 84 in the sewage system and 14 in parks, during the winter of 2016-17 in a rodent surveillance and control program in Barcelona (permission no. SF/044 obtained from the regional government of Catalonia). We treated the rats according to Directive 2010/63/EU of the European Parliament and Council decision of September 22, 2010. We obtained DNA from $10 \mathrm{mg}$ of spleen using the Purification of Total DNA Kit (QIAGEN, https://www.qiagen.com), following the manufacturer's instructions. We processed the samples whose DNA concentration was too low with the extraction kit by the phenol-chloroform-isoamyl $(25: 24: 1)$ DNA extraction technique. We quantified the parasite DNA by qPCR using Taqman probe with Fam fluorochrome (4).

Only 1 rat $(7.1 \%)$ captured in the parks tested positive for $L$. infantum. However, rats captured in the sewage system showed a $33.3 \%$ prevalence $(28 / 84)$ of $L$. infantum infection. The estimated number of parasites in the positive samples from spleens varied considerably, ranging from 0.28 to $>2,200$ (Table). Histologic sections of positive spleens were stained by Giemsa and by the streptavidin-biotin peroxidase complex immunohistochemical method (5) (Appendix Figure, https://wwwnc.cdc.gov/ EID/article/25/6/18-1027-App1.pdf).

The low number of infected Norway rats found in Mediterranean countries in Europe so far has led to the species being categorized as an incidental host, capable of becoming infected but considered irrelevant to the longterm persistence of the disease (2,6-8). However, our study demonstrates the importance of the trapping site for finding a large Leishmania-infected rat population.

According to the World Health Organization, the incrimination of a particular mammal as a Leishmania reservoir must depend on an accumulation of evidence (9). First, the reservoir must be sufficiently abundant 
Table. Real-time PCR results for the 29 Leishmania infantumpositive Norway rats analyzed, Barcelona, Spain*

\begin{tabular}{|c|c|c|c|}
\hline Rat no. & $\mathrm{C}_{\mathrm{t}} \dagger$ & Estimated no. parasites & SD \\
\hline 1 & 32.81 & 75.67 & 25.70 \\
\hline 2 & 32.33 & 50.68 & 12.21 \\
\hline 3 & 29.25 & 723.15 & 345.67 \\
\hline 4 & 30.84 & 344.30 & 56.40 \\
\hline 5 & 38.50 & 0.28 & 0.20 \\
\hline 6 & 28.49 & 10.81 & 3.36 \\
\hline 7 & 28.46 & $2,272.46$ & 771.72 \\
\hline 8 & 32.32 & 118.12 & 63.76 \\
\hline 9 & 29.60 & 516.20 & 199.36 \\
\hline 10 & 28.10 & 14.24 & 2.98 \\
\hline 11 & 30.19 & 566.28 & 7.32 \\
\hline 12 & 28.41 & 11.98 & 5.09 \\
\hline 13 & 29.96 & 679.46 & 35.59 \\
\hline 14 & 31.47 & 31.38 & 0.20 \\
\hline 15 & 31.46 & 215.66 & 66.84 \\
\hline 16 & 30.79 & 356.32 & 42.03 \\
\hline 17 & 33.01 & 66.31 & 31.43 \\
\hline 18 & 29.68 & 465.21 & 68.73 \\
\hline 19 & 30.75 & 370.40 & 70.72 \\
\hline 20 & 31.60 & 1.44 & 1.51 \\
\hline 21 & 26.52 & 48.81 & 20.15 \\
\hline 22 & 31.82 & 159.43 & 27.73 \\
\hline 23 & 28.15 & 0.33 & 17.66 \\
\hline 24 & 28.56 & 9.98 & 1.30 \\
\hline 25 & 30.25 & 547.67 & 93.15 \\
\hline 26 & 30.93 & 161.73 & 11.80 \\
\hline 27 & 28.38 & 11.59 & 2.70 \\
\hline 28 & 29.84 & 761.38 & 223.52 \\
\hline 29 & 33.16 & 55.22 & 1.41 \\
\hline
\end{tabular}

and long-lived. In this sense, the Norway rat is the most abundant mammal in cities, with a lifespan of around 1-3 years. The lack of predators or interspecific competition in cities guarantees a longer lifetime than in wild environments. Second, intense host-sand fly contact is necessary. Sewers are a breeding site for Phlebotomus sand flies, which can reach abundant population levels (10). Third, the prevalence of Leishmania infection should be $>20 \%$. Our study revealed a $33.3 \%$ prevalence in Norway rats in the sewers. Fourth, the course of infection should be nonpathogenic and long enough to enable the parasites to survive any nontransmission season. Neither splenomegaly nor hepatomegaly were evident in the affected animals, although further studies should be addressed to assess the true pathogenic degree of rat leishmaniasis. Finally, parasites should be available in the skin or the blood in sufficient numbers to be taken up by a sand fly. The availability of the parasite to the sand fly cannot be proved by our findings. However, in naturally infected Norway rats in Spain, L. infantum was detected in the hair of the rats by molecular methods (7).

Only counting sewer rats, and not those living above ground, a 0.13 rat-per-person scenario is suspected for Barcelona (Agència de Salut Pública de Barcelona, pers. comm., June 2018). Therefore, the prevalence found in the sewers in this study means that there could be $>70,000$ underground rats with leishmaniasis in Barcelona $(1,620,809$ human inhabitants in 2017), a figure of public health concern for a potential reservoir.

If future xenodiagnostic studies determine that sand flies become infected successfully by the rats, as has already been demonstrated in the case of its congener, the black rat, ( $R$. rattus) (11), the finding of a new reservoir, besides the dog, would be a major advance in the epidemiology of leishmaniasis. However, the complexity of rat control would represent a great hindrance to overcome.

\section{About the Author}

Dr. Galán-Puchades is director of the Parasites and Health Research Group of the University of Valencia, BurjassotValencia, Spain. Her research interests include neglected parasitic diseases.

\section{References}

1. Miró G, López-Vélez R. Clinical management of canine leishmaniosis versus human leishmaniasis due to Leishmania infantum: putting "One Health" principles into practice. Vet Parasitol. 2018;254:151-9. http://dx.doi.org/10.1016/ j.vetpar.2018.03.002

2. Millán J, Ferroglio E, Solano-Gallego L. Role of wildlife in the epidemiology of Leishmania infantum infection in Europe. Parasitol Res. 2014;113:2005-14. http://dx.doi.org/10.1007/ s00436-014-3929-2

3. Riera C, Napp S, Manzanares S. Epidemiology of human leishmaniasis in the city of Barcelona (1997-2014) [in Spanish]. Rev Enf Emerg. 2016;15:68-76.

4. Francino O, Altet L, Sánchez-Robert E, Rodriguez A, Solano-Gallego L, Alberola J, et al. Advantages of real-time PCR assay for diagnosis and monitoring of canine leishmaniosis. Vet Parasitol. 2006;137:214-21. http://dx.doi.org/10.1016/ j.vetpar.2006.01.011

5. Tafuri WL, Santos RL, Arantes RM, Gonçalves R, de Melo MN, Michalick MS, et al. An alternative immunohistochemical method for detecting Leishmania amastigotes in paraffin-embedded canine tissues. J Immunol Methods. 2004;292:17-23. http://dx.doi.org/ 10.1016/j.jim.2004.05.009

6. Psaroulaki A, Antoniou M, Toumazos P, Mazeris A, Ioannou I, Chochlakis D, et al. Rats as indicators of the presence and dispersal of six zoonotic microbial agents in Cyprus, an island ecosystem: a seroepidemiological study. Trans R Soc Trop Med Hyg. 2010;104:733-9. http://dx.doi.org/10.1016/ j.trstmh.2010.08.005

7. Muñoz-Madrid R, Belinchón-Lorenzo S, Iniesta V, Fernández-Cotrina J, Parejo JC, Serrano FJ, et al. First detection of Leishmania infantum kinetoplast DNA in hair of wild mammals: application of qPCR method to determine potential parasite reservoirs. Acta Trop. 2013;128:706-9. http://dx.doi.org/ 10.1016/j.actatropica.2013.08.009

8. Tsakmakidis I, Angelopoulou K, Dovas CI, Dokianakis E, Tamvakis A, Symeonidou I, et al. Leishmania infection in rodents in Greece. Trop Med Int Health. 2017;22:1523-32. http://dx.doi.org/10.1111/tmi.12982

9. World Health Organization. Control of the leishmaniases. WHO Technical Report Series no. 949. Geneva: The Organization; 2010. 
10. Lucientes J, Castillo JA, Gracia MJ, Perbáñez A. Phlebotomus, from the biology to the control [in Spanish]. REDVET. 2005;6 [cited 2018 May 16]. http://www.veterinaria.org/revistas/redvet/ n080805.html

11. Gradoni L, Pozio E, Gramiccia M, Maroli M, Bettini S. Leishmaniasis in Tuscany (Italy): VII. Studies on the role of the black rat, Rattus rattus, in the epidemiology of visceral leishmaniasis. Trans R Soc Trop Med Hyg. 1983;77:427-31. http://dx.doi.org/10.1016/0035-9203(83)90102-5

Address for correspondence: Maria Teresa Galán-Puchades, University of Valencia, Department of Parasitology, Faculty of Pharmacy, Av Vicent Andrés Estellés s/n, 46100 Burjassot-Valencia, Spain; email: mteresa. galan@uv.es

\section{Influenza D Virus Infection in Dromedary Camels, Ethiopia}

\author{
Shin Murakami, Tomoha Odagiri, \\ Simenew Keskes Melaku, Boldbaatar \\ Bazartseren, Hiroho Ishida, \\ Akiko Takenaka-Uema, Yasushi Muraki, \\ Hiroshi Sentsui, Taisuke Horimoto
}

Author affiliations: University of Tokyo, Tokyo, Japan

(S. Murakami, T. Odagiri, H. Ishida, A. Takenaka-Uema, T. Horimoto); Addis Ababa Science and Technology University, Addis Ababa, Ethiopia (S.K. Melaku); Institute of Veterinary Medicine, Ulaanbaatar, Mongolia (B. Bazartseren); Iwate Medical University, Iwate, Japan (Y. Muraki); Nihon University, Kanagawa, Japan (H. Sentsui)

DOI: https://doi.org/10.3201/eid2506.181158

Influenza D virus has been found to cause respiratory diseases in livestock. We surveyed healthy dromedary camels in Ethiopia and found a high seroprevalence for this virus, in contrast to animals co-existing with the camels. Our observation implies that dromedary camels may play an important role in the circulation of influenza D virus.

I nfluenza D virus (IDV) was first isolated from pigs with respiratory symptoms in the United States in 2011 (1). Epidemiologic analyses revealed that the most likely main host of IDV is cattle, because the seropositivity rate in these animals is higher than that for other livestock (2-4). In a recent report, dromedary camels (Camelus dromedaries) exhibited substantially high seroprevalence (99\%) for IDV in
Kenya (5), suggesting that this animal is a potential reservoir of IDV. We examined seroprevalence of IDV in dromedary camels in Ethiopia and in Bactrian camels (Camelus bactrianus) in Mongolia.

We collected serum samples from dromedary camels $(\mathrm{n}=38$; average age 4.3 years, range $1-13$ years), goats ( $\mathrm{n}$ $=20$; average age 3.9 years, range $1-8$ years), sheep $(\mathrm{n}=$ 20 ; average age 2.7 years, range $1-4$ years), cattle ( $n=15$; average age 6.7 years, range, $1-11$ years), and donkeys (n $=2$; ages 1 and 6) from 2 herds in Bati district, Amhara region, and 1 herd in Fafen district, Somali region, Ethiopia. All animals were apparently healthy, shared the same pasturage during the day, and stayed in barns specific for each animal species at night. To detect influenza D infection, we titrated the serum samples by hemagglutination inhibition (HI) assay using 3 antigenically distinct influenza D strains: D/swine/Oklahoma/1334/2011 (D-OK lineage; D/OK) (1), D/bovine/Nebraska/9-5/2013 (D/660-lineage; $\mathrm{D} / \mathrm{NE}$ ) (6), and D/bovine/Yamagata/10710/2016 (D/Japan-lineage; D/Yamagata) (7). For the HI test, we treated the samples with receptor-destroying enzyme (RDEII; Denka Seiken, http://www.keyscientific.com) at $37^{\circ} \mathrm{C}$ for $16 \mathrm{~h}$, followed by heat inactivation at $56^{\circ} \mathrm{C}$ for $30 \mathrm{~min}$. We then reacted serially diluted samples with each virus (4 HAU) at room temperature for $30 \mathrm{~min}$ and incubated them with a $0.6 \%$ suspension of turkey red blood cells at room temperature for $30 \mathrm{~min}$. The HI titer of each sample was expressed as the reciprocal of the highest sample dilution that completely inhibited HA. We considered samples with HI titer $\geq 1: 40$ positive, to eliminate nonspecific reactions at low dilutions $(4,8,9)$.

Of the 21 dromedary camel samples from Bati, 10 were positive for $\mathrm{D} / \mathrm{OK}, 11$ for $\mathrm{D} / \mathrm{NE}$, and 19 for $\mathrm{D} / \mathrm{Ya}-$ magata (Figure). Of other animal samples, only 1 goat sample was positive (titer 1:40), indicating that the prevalence rate of influenza $\mathrm{D}$ antibodies was higher in dromedary camels than in co-grazing ruminants in the tested herd. The data on the camels' age indicated that the HI antibodies were not detected due to maternal antibodies, which is only stable for 5-6 months in dromedary camels (10). Much closer face-to-face contact may be required for virus transmission among different animal species. The HI titers in camel samples were higher for D/Yamagata (range 1:40-1:160) than those for D/OK and $\mathrm{D} / \mathrm{NE}$ (1:40-1:80). Meanwhile, we found several positives in dromedary camel samples from Fafen, albeit at lower positive rates and titers compared with those in Bati (Figure).

We confirmed the specificity of the HI reaction with a viral neutralizing test using HI-positive samples (data not shown). HI titers obtained were not high, suggesting that the infections may have occurred in these animals some time ago or that results might have been due to the variance of $\mathrm{HI}$ 